\title{
Interaction between Current Imbalance and Magnetization in LHC Cables
}

\author{
M. Haverkamp, A. Kuijper, A. den Ouden, B. ten Haken, L. Bottura and H. H. J. ten Kate
}

\begin{abstract}
The quality of the magnetic field in superconducting accelerator magnets is associated with the properties of the superconducting cable. Current imbalances due to coupling currents $\Delta I$, as large as $100 \mathrm{~A}$, are induced by spatial variations of the field sweep rate and contact resistances. During injection at a constant field all magnetic field components show a decay behavior. The decay is caused by a diffusion of coupling currents into the whole magnet. This results in a redistribution of the transport current among the strands and causes a demagnetization of the superconducting cable. As soon as the field is ramped up again after the end of injection, the magnetization rapidly recovers from the decay and follows the course of the original hysteresis curve. In order to clarify the interactions between the changes in current and magnetization during injection we performed a number of experiments. A magnetic field with a spatially periodic pattern was applied to a superconducting wire in order to simulate the coupling behavior in a magnet. This model system was placed into a stand for magnetization measurements and the influence of different powering conditions was analyzed.
\end{abstract}

Index Terms-Field component decay, Magnetization measurements, Snapback, Superconducting accelerator magnets.

\section{INTRODUCTION}

\section{A. Field Errors in the LHC Magnets}

In the Large Hadron Collider (LHC) a very precise control of the beam parameters has to be provided in order to accelerate protons from injection to an energy of $7 \mathrm{TeV}$ [1]-[3]. Apart from geometric field errors, the saturation of the iron yoke and eddy currents, additional field imperfections in the superconducting magnets are caused by the $\mathrm{AC}$ and $\mathrm{DC}$ diamagnetic properties of the superconducting material in the coil. The LHC magnets are wound from flat Rutherford cables, consisting of 28 strands in the inner layer and 36 strands in the outer layer. The inner layer strands are twisted from about 8800 , the outer layer strands from about 6400 superconducting NbTi-filaments. Their contribution to the quality of the magnetic field can be especially important at the low injection field level of $0.535 \mathrm{~T}$. This contribution is often referred to as persistent currents, due to the long lasting shielding currents, which are responsible for the diamagnetic beha-

Manuscript received September 17, 2000. This research is supported by the Technology Foundation STW, applied science division of NOW and the technology programme of the Ministry of Economic affairs.

M. Haverkamp, A. Kuijper, A. den Ouden, B. ten Haken and H. H. J. ten Kate are with the University of Twente TN / LT, P.O. Box 217, 7500 AE Enschede, The Netherlands.

M. Haverkamp, L. Bottura and H. H. J. ten Kate are with CERN, 1211 Geneva 23, Switzerland.

(e-mail: Markus.Haverkamp@ @ern.ch, telephone: 0041-22-7678381). vior of the filaments. The LHC will also have to deal with another class of effects, which are associated as well with the properties of the superconducting cable. During long periods of constant current excitation, especially during injection, all components of the magnetic field decay significantly.

\section{B. The Field Component Decay}

Two different contributions to the field decay were identified. The first one is caused by flux creep and decays quite fast, with a logarithmic time dependence. At $2 \mathrm{~K}$ it is typically in the range of $1 \%$ of the original magnetization [4] and represents roughly 20 to $80 \%$ of the decay during injection, depending on interstrand resistances and other parameters of the magnet [1]. The second contribution, however, exhibits a long lasting decay and only appears if the magnet was precycled with a high current. The source of the second decay component can be found in "current imbalances" in the Rutherford cable, where different strands take different fractions of the cable transport current. Thus, coupling currents $\Delta \mathrm{I}$, as large as $100 \mathrm{~A}$ [5], circulate in various loops formed by strand sections and interstrand contacts, with different time constants up to several hours and over different distances, only limited by the length of the cable. Obviously the average transport current in the cable is not affected. These so-called Boundary Induced Coupling Currents (BICCs) follow the zigzag path of the strands in the cable and can be observed as a spatially periodic pattern in all field harmonics. One period of this modulation is equal to the cable twist pitch. During a ramp, BICCs are induced especially in regions where the cable is exposed to large variations in the field sweep rate and contact resistances [6], [7]. As long as the external field sweeps, the spatially periodic pattern does not affect the magnetization. However, during periods of constant magnetic field, as for example during injection, the field component decay is caused by a diffusion of BICCs along the cable and thus into the whole magnet. This results in a redistribution of the transport current among the strands and generates a spatially periodic change of the internal field along the cable. This periodic change, thus, modifies the shielding currents in the filaments. In those parts of the filaments where the induced field is parallel to the background field, the superconductor follows the up-ramp branch of the hysteresis curve. In the parts with antiparallel induced field, however, a new layer of shielding currents with opposite polarity is induced. This causes a spatial modulation in magnetization. As a net effect a decrease of the average cable magnetization, typically in the range of $2 \%$, and a change in all multipoles are observed [8]. In order to reduce the average magnetization inside the cable by $2 \%$, the amplitude of the periodic field modulation due to the redistribution 
of the BICCs has to change in the range of $0.6 \mathrm{mT}$. Thus, already a change in BICCs of $6 \mathrm{~A}$ in the cable can cause a decay.

\section{The Snapback Effect}

As soon as, after the end of injection, the external field is ramped up again, the shell of shielding currents in the filaments is cancelled and the magnetization follows the up-ramp branch of the original hysteresis curve. This "snapback" occurs during a field change of typically $15-20 \mathrm{mT}$.

\section{EXPERIMENT}
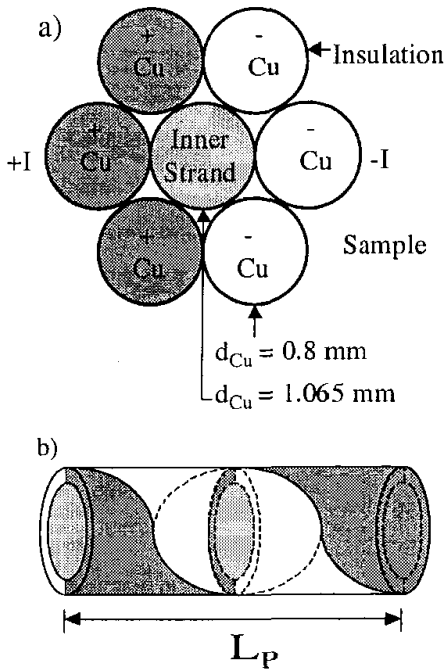

Fig. 1. a) Cross section of the test cable, wound from an LHC inner strand and 6 copper wires. b) Sideview of the cable, showing central strand and twisted current shell.

A new experiment is being developed in order to study the interaction between current redistribution and magnetization during decay and snapback and to verify the theory described in [8]. A cable is twisted from an LHC inner strand and six surrounding insulated copper wires (Fig. 1) and wound around a sample holder. The twisted copper wires are used as a current shell, in order to induce a dipole field with a spatially periodic modulation into the central strand of the cable. Along the length of the strand the field induced by a current in the copper wires changes its angle with respect to the original strand magnetization. During the experiment the LHC strand is first fully magnetized in an external field. If a current is applied to the copper wires, the periodic field modulation induces a decay of the filament magnetization. As soon as the external field is increased the snapback of the magnetization is visible. The magnetization measurement is performed inside a cylindrical cryostat (Fig. 2). The sample is placed be-

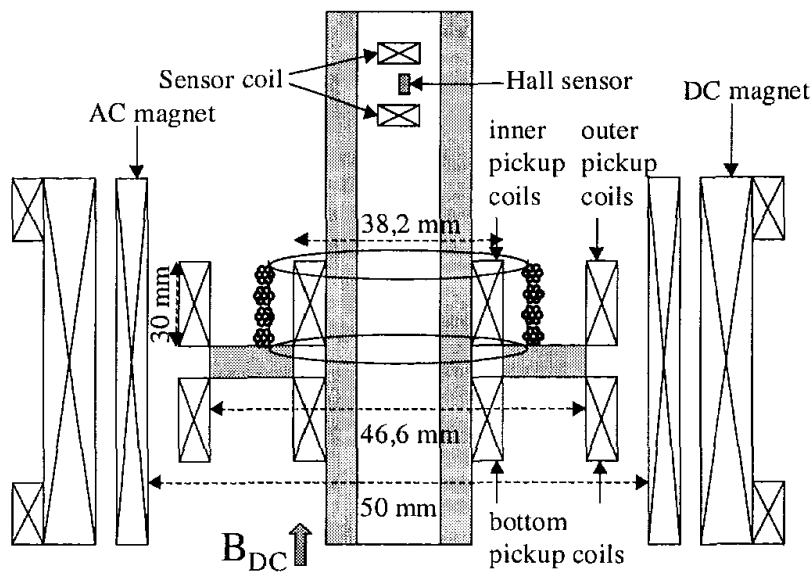

Fig. 2. Setup for the measurement of magnetization.

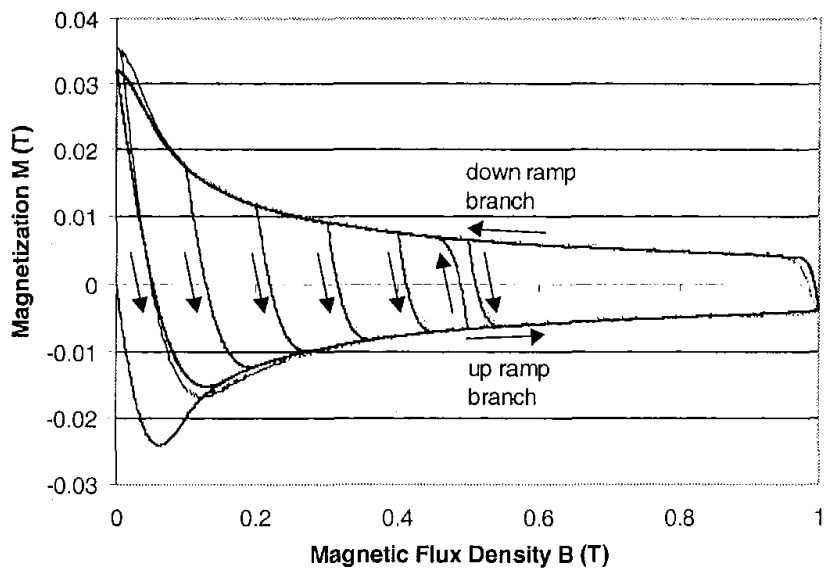

Fig. 3. Calculated (thick) and measured (thin) hysteresis curves of the LHC strand.

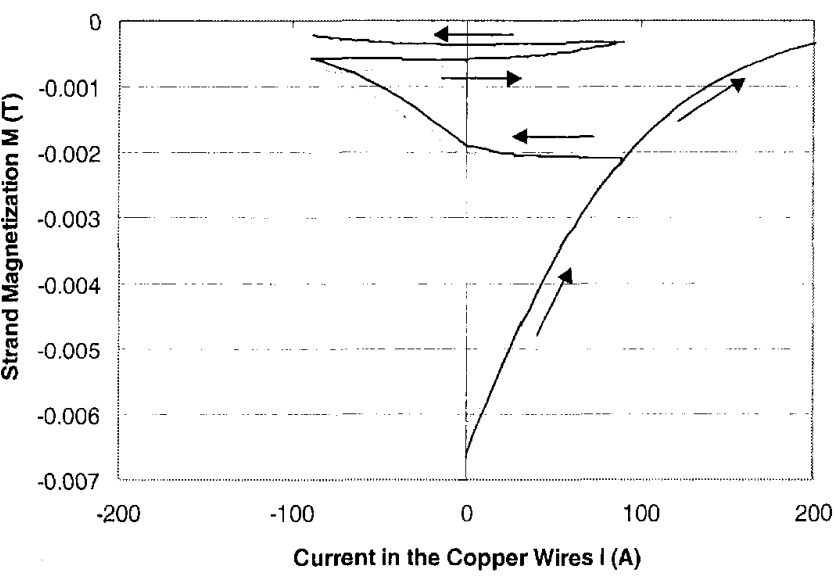

Fig. 4. Magnetization of the sample as a function of the current in the copper wires (thick: calculated and thin: measured values).

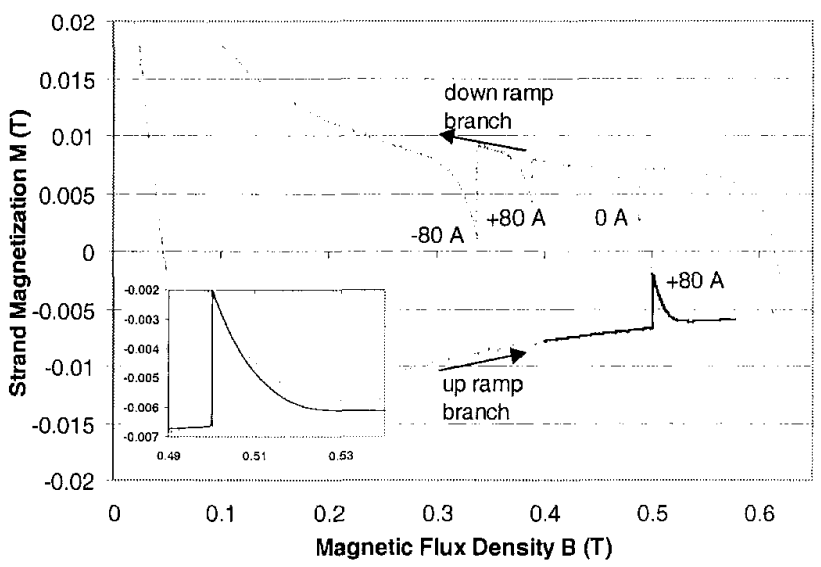

Fig. 5. Thin: Measured hysteresis curve showing decays and snapbacks. Thick: A decay and snapback was calculated between 0.4 and $0.6 \mathrm{~T}$. The insert shows a magnification.

tween two concentric superconducting pickup coils. The inner coil is used to increase the homogeneity of the mutual induction between the sample and the pickup set. Two additional bottom coils compensate the applied field. The magnetic moment of the sample can be obtained by integrating the induced 
voltage over time. The pickup coils are connected in series in a superconducting circuit to a sensor coil. In our setup the circuit acts as a superconducting integrator. Thus, the magnetic field inside the sensor coil is proportional to the magnetization signal and can be read out by a Hall sensor. AC and DC external fields can be applied to the sample, when powering the AC and DC magnets, which are also shown in Fig. 2. Details on the measurement apparatus can be found in [9]. The experiment is performed at $4.2 \mathrm{~K}$, in a liquid Helium bath at atmospheric pressure. The reproducibility of the measurements is better than $10 \%$.

\section{EXPERIMENTAL RESULTS}

\section{A. Measured Hysteresis Curves}

In order to verify the measurement capability and to characterize the strand, the external field is cycled between different minima and always the same maximum. We measure hysteresis curves of the sample, without applying any current to the copper wires. The measurements are shown in Fig. 3 (thin curve). Especially for low fields one should expect an influence of the local field profile in the filaments on the critical current density. Besides, filament bridging and the critical field $B c_{l}$ of the Meissner phase should affect the magnetization. $B c_{l}$ is about $11 \mathrm{mT}$ for $\mathrm{NbTi}$ (at $4.2 \mathrm{~K}$ ). At low field, these effects make it especially difficult to measure the $J C(B)$ dependence of the superconductor.

\section{B. Demonstration of the Demagnetization Effect}

To demonstrate the interaction between the current redistribution and magnetization, the strand is first fully magnetized in a constant external field of $0.5 \mathrm{~T}$. Then the sample magnetization is measured as a function of the current in the copper wires. In Fig. 4 the experimental data is shown (thin curve). A current in the twisted copper wires demagnetizes those parts of the strand, where the induced field opposes the background field. Here, the magnetization follows the down-ramp branch. In the parts of the cable where the current loops of the wire generate a field parallel to the background field, the magnetization follows the up-ramp branch. The net effect for the entire cable is a demagnetization. For small currents every additional Ampere decreases the magnetization by about $0.05 \mathrm{~m} \mathrm{~T}$. For higher currents (above $50 \mathrm{~A}$ ) a saturation behavior is clearly visible. After reaching a maximum of $90 \mathrm{~A}$, the current is reduced again. As a consequence, the fully magnetized half twist pitches of the cable are demagnetized and the previously demagnetized ones are magnetized and saturated again. The average magnetization remains constant till $0 \mathrm{~A}$ are reached. A current of opposite polarity further reduced the magnetization of the presently demagnetized half twist pitches and leaves the saturated ones constant. The average sample magnetization is further reduced, but with a considerably smaller slope. After reaching $-90 \mathrm{~A}$, the current is decreased another time, and once again the magnetization remains constant. Repeated measurements have shown that with every new increase of the current the slope gets smaller and smaller. However, in order to give a more precise and quantitative explanation, one has to take into account that the twisted copper wires create a field, which changes direction along the strand. A demagnetization behavior quite similar to these measurements has been observed in the HERA correction windings [10].

\section{Demonstration of the Decay and Snapback Effect}

Fig. 5 (thin lines) shows a measured hysteresis curve, where the ramp is stopped at $0.5 \mathrm{~T}$ (see also magnification in the insert). During a period of constant background field the current in the copper wires is increased from 0 to $80 \mathrm{~A}$. A demagnetization can be seen very clearly. When ramping up the external field again, the demagnetization is cancelled and the magnetization "snaps back" to the hysteresis curve. With respect to the original hysteresis curve, the one after the snapback is shifted upwards by a constant offset. This offset is due to a residual current loop of the copper wires. On the downramp branch of the hysteresis curve snapbacks are performed using demagnetization currents of different polarities. The shifts after the snapbacks are proportional to the currents applied.

\section{Calculations}

In order to calculate the penetration of magnetic flux into a superconducting filament we use an algorithm based on the iteration of an integral equation [11]. It describes the distribution of shielding currents due to a penetrating perpendicular field. The algorithm is valid for flux densities $B$ and critical sheet current densities $J d$, which are larger than $B c_{l}$. Let's assume a cylindric filament, parallel to the $\mathrm{z}$-axis of a coordinate system, with a circular cross section $S$ in the $x-y$-plane. From the Maxwell equations one can derive a formula for the distribution of shielding currents $\mathrm{J}$ :

$J(\vec{r}, t)=\frac{-1}{\mu_{0}} \int_{S} Q^{-1}\left(\vec{r}, \vec{r}^{\prime}\right)\left[A\left(\vec{r}^{\prime}, t\right)+x^{\prime} B_{y}-y^{\prime} B_{x}\right] d S^{\prime}$.

$\mathrm{Q}^{-1}$ is the inverse of the integrator Kernel $\mathrm{Q}$ from the solution of the Laplace equation:

$Q\left(\vec{r}, \vec{r}^{\prime}\right)=\frac{1}{2 \pi} \ln \left|\vec{r}-\vec{r}^{\prime}\right|$.

The penetrating field $\left(B_{x}, B_{y}\right)$ is perpendicular to the filament and also $\vec{r}=(x, y, 0)$ and $\vec{r}^{\prime}=\left(x^{\prime}, y^{\prime}, 0\right)$ are vectors in the $x$ $\mathrm{y}$-plane. The vector potential A has only a component in z-direction and is calculated as a time integral of the electric field:

$A=\int_{0}^{t} E(t) d t$.

The electric field can be expressed as a function of $\mathrm{J}$ and $\mathrm{B}$ :

$E(J, B)=\rho(J, B) J$.

Here, $\rho$ represents the resistivity:

$\rho(J, B)=r_{0} B\left(J / J_{c}\right)^{\sigma} /\left(1+\left(J / J_{c}\right)^{\sigma}\right)$.

The creep exponent $\sigma$ describes ohmic behavior for $\sigma=0$ and the Bean model for $\sigma=\infty$. For LHC strands $\sigma$ is typically in the range of 40 (at $4.2 \mathrm{~K}$ and $7 \mathrm{~T}$ ). The flux flow resistivity prefactor $r_{0}$ is a constant. See [11] for more details on the model. 


\section{A. Calculated Hysteresis Curves}

The model above takes into account the dependence of the critical current density on the local field in the cross section of the filament. A comparison between calculated (thick) and measured (thin) data (Fig. 3) allows to more precisely determine the $J c(B)$ dependence, using iterative approximations. The hysteresis curves, calculated using the best $J c(B)$ fit, accurately describe the experimental data. Below $0.2 \mathrm{~T}$ small deviations are still visible, possibly due to filament bridging and due to a non-zero value of $B c_{l}$. Hysteresis curves were also calculated for different minimum fields. The penetration phases of the measured curves are slightly larger, which could be due to deviations of the filament cross sections from a circular shape. At a field level of about $0.5 \mathrm{~T}$ the effect of a field increase and decrease was calculated. The increase follows the original hysteresis curve and hardly changes the magnetization. The filaments are already saturated and the curve follows the $J_{C}(B)$ dependence. A field decrease, on the other hand, follows the down-ramp branch. Here, a new current shell of opposite polarity is induced into the filaments. As a consequence the magnetization decreases very rapidly.

\section{B. Calculation of the Demagnetization Effect}

The field change due to the current in the copper wires alters its direction along the strand, with respect to the initial magnetization of the fully penetrated filaments. In order to take this effect into account, calculations were performed with field changes of equal magnitudes and at 24 different angles with respect to the original magnetization. The thick curves in Fig. 4 show the average of these 24 different calculations as a function of the amplitude of the field change. In order to be able to make comparisons with the experimental data, we assume that every Ampere of current in the copper wires induces a field of $0.245 \mathrm{mT}$, which is quite close to the expected value. For increasing current a demagnetization and a saturation behavior is visible and the simulations very well describe the experimental results. In a second calculation the increase of the demagnetizing field is stopped at $22 \mathrm{mT}$, which corresponds to 90 A of current in the copper wires. Afterwards the demagnetizing field was cycled back and forth several times, between $+22 \mathrm{mT}$ and $-22 \mathrm{mT}$. The simulations qualitatively show the same behavior as the experimental data, but deviate more and more with every cycle.

\section{Calculation of the Decay and Snapback Effect}

For the same 24 angles as mentioned previously, the demagnetization and a subsequent ramp of the external field were calculated. In Fig. 5 the simulated snapback (thick curve) is shown for a field range between 0.4 and $0.6 \mathrm{~T}$, and plotted together with the measured data (thin curve). A magnification is displayed in the insert. The calculated demagnetization amplitudes and snapback phases coincide with the experimental data. A slight difference in the phases can be explained by the slightly larger penetration phases of the measured hysteresis curves, which are probably due to deviations of the filament cross sections from a circular shape. The shift between the calculated and measured snapback is due to a residual current loop, as already explained before.

\section{Conclusions}

Measured and calculated hysteresis curves of an LHC strand are compared. Taking into account the $J_{C}(B)$ dependence on the local field in the cross section of the filaments, it is possible to calculate hysteresis curves, which correctly describe the experimental data. Significant deviations are only found for very low fields. They are probably due to filament bridging or the influence of $B c_{l}$.

The magnetization of the sample is measured as a function of the current in the copper wires and the interaction between current redistribution and strand magnetization is confirmed. Also in this case we are able to explain our observations quantitatively.

Finally, the snapback effect is demonstrated experimentally. Apart from a drift due to a residual current loop, measurements and calculations correspond.

These measurements are the first experimental proof of the theory described in [8]. Apart from that, for the first time it is possible to correctly calculate the effects.

The future goal will be to derive formulas for the compensation of decay and snapback in the real LHC accelerator.

\section{ACKNOWLEDGMENT}

The authors would like to thank A. Akhmetov, E. Brandt, L. Krempasky, J. Rabbers, C. Schmidt, H. Steffens, W. Venturini, A. Verweij, S. Wessel and R. Wolf for many good ideas, supplied materials, software, data, manual skills and constant encouragement.

\section{REFERENCES}

[1] M. Schneider, "Decay and Snapback Studies on LHC Dipole Model Magnets: A Scaling Law", PhD thesis, TU Wien, 1998.

[2] L. Bottura, M. Schneider and M. Haverkamp, "Long Term Dynamic Effects in LHC Dipole Magnets", unpublished.

[3] M. Haverkamp, L. Bottura and M. Schneider, "Studies of Decay and Snapback Effects on LHC Dipole Magnets", Int. Phys. Conf. Ser., 167, pp. 1183-1186, 2000.

[4] S. Le Naour et al, "Magnetization Measurements on LHC Superconducting Strands", IEEE Trans. Appl. Sup. 9 2, pt. 2, pp. 1763-1766, 1999.

[5] L. Bottura, L. Walkiers and Z. Ang, "Experimental Evidence of Boundary Induced Coupling Currents in LHC Prototypes", IEEE Trans. Appl. Sup., vol. 7 (2), pp. 801-804, 1997.

[6] A. P. Verweij, "Electrodynamics of Superconducting Cables in Accelerator Magnets", PhD Thesis, Twente University, The Netherlands, 1995.

[7] L. Krempasky and C. Schmidt, "Experimental Verification of 'Supercurrents' in Superconducting Cables exposed to AC-Fields", Cryogenics, 39, pp. 23-33, 1999.

[8] R. Wolf, "The Decay of the Field Integral in Superconducting Accelerator Magnets wound with Rutherford Cables", Proc. of $15^{\text {th }}$ Int. Mag. Tech. Conf,, Beijing, pp. 238-241, Science Press, 1998.

[9] M. Woudstra, "Electromagnetic Characterisation of BSCOO-2212 Multi Filament Wires", Graduation Report, University of Twente, The Netherlands, 1995.

[10] M. Pekeler et al., "Coupled Persistent-Current Effects in the HERA Dipoles and Beam Pipe Correction Coils", DESY HERA 92-06, April, 1992.

[11] E. H. Brandt, "Superconductors of Finite Thickness in a Perpendicular Magnetic Field: Strips and Slabs", Physical review B, Vol 54, number 6, pp. 4246-4263, 1 August 1996 II. 